\title{
Continuity of the Bessel wavelet transform on certain Beurling-type function spaces
}

\author{
Akhilesh Prasad $^{1}$, Ashutosh Mahato ${ }^{1 *}$ and MM Dixit ${ }^{2}$
}

\section{"Correspondence:}

ashumahato@gmail.com

'Department of Applied

Mathematics, Indian School of

Mines, Dhanbad, 826004, India

Full list of author information is

available at the end of the article

\begin{abstract}
In this paper, continuity of the Bessel wavelet transform of a suitable function $\phi$ in terms of an appropriate mother wavelet $\psi$ is investigated on certain Beurling-type function spaces.
\end{abstract}

MSC: $46 \mathrm{~F} 12 ; 26 \mathrm{~A} 33$

Keywords: Bessel wavelet transform; Hankel transform; Hankel translation; Zemanian space

\section{Introduction}

Bessel wavelet transforms have applications in the study of boundary value problems on the half-line. The Hankel transform and pseudo-differential operators associated with the Bessel operator have been studied on some Beurling-type function spaces by [1,2] and [3] respectively. The aim of the present paper is to study the continuity of the Bessel wavelet transform on certain Beurling-type function spaces.

Let us define $L_{\mu, p}\left(\mathbb{R}_{+}\right), 1 \leq p<\infty$, as the space of those real measurable functions $\phi$ on $\mathbb{R}_{+}=(0, \infty)$ for which

$$
\begin{aligned}
& \|\phi\|_{L_{\mu}, p}=\left[\int_{0}^{\infty}|\phi(x)|^{p} d \mu(x)\right]^{1 / p}<\infty, \quad 1 \leq p<\infty, \\
& \|\phi\|_{L_{\mu}, \infty}=\underset{0<x<\infty}{\operatorname{ess} \sup }|\phi(x)|<\infty,
\end{aligned}
$$

where $d \mu(x)=1 / 2^{\mu} \Gamma(\mu+1) x^{2 \mu+1} d x$.

From $[1,4]$, the Hankel translation $\phi \in L_{\mu, p}\left(\mathbb{R}_{+}\right)$is defined by

$$
\tau_{y} \phi(x):=\phi(x, y):=\int_{0}^{\infty} \phi(z) D(x, y, z) d \mu(z), \quad 0<x, y<\infty,
$$

where

$$
D(x, y, z):=\int_{0}^{\infty} j(x t) j(y t) j(z t) d \mu(t)
$$

with $j(x)=2^{\mu} \Gamma(\mu+1) x^{-\mu} J_{\mu}(x)$.

From [5], in terms of the Hankel translation $\tau_{y}$ and dilation $D_{a}$ defined by $D_{a} \phi(x, y):=$ $\phi(x / a, y / a)$, we define the Bessel wavelet $\theta_{b, a}$ by

$$
\theta_{b, a}(x):=D_{a} \tau_{b} \theta(x)=D_{a} \theta(b, x)=\theta(b / a, x / a):=\int_{0}^{\infty} D(b / a, x / a, z) \theta(z) d \mu(z) .
$$


Let $\theta \in L_{\mu, p}\left(\mathbb{R}_{+}\right)$, then the Bessel wavelet transform of $g \in L_{\mu, q}\left(\mathbb{R}_{+}\right),(1 / p+1 / q=1)$, is defined by

$$
\begin{aligned}
\left(B_{\theta} g\right)(b, a)= & \left\langle g(t), \theta_{b, a}(t)\right\rangle=\int_{0}^{\infty} g(t) \overline{\theta_{b, a}(t)} d \mu(t) \\
= & \int_{0}^{\infty} \int_{0}^{\infty} g(t) \overline{\theta(z)} D(b / a, t / a, z) d \mu(z) d \mu(t) \\
= & \int_{0}^{\infty}(b x / a)^{1 / 2} J_{\mu}(b x / a) x^{-\mu-1 / 2} b^{-\mu-1 / 2} a^{2 \mu+1} \\
& \times\left\{\int_{0}^{\infty} t^{\mu+1 / 2} g(t)(t x / a)^{1 / 2} J_{\mu}(t x / a) d t\right. \\
& \left.\times \int_{0}^{\infty} z^{\mu+1 / 2} \overline{\theta(z)}(z x)^{1 / 2} J_{\mu}(z x) d z\right\} d x .
\end{aligned}
$$

Now, using the Hankel transformation,

$$
\left(h_{\mu} \phi\right)(u)=\int_{0}^{\infty}(x u)^{1 / 2} J_{\mu}(x u) \phi(x) d x, \quad \mu \geq-1 / 2
$$

which is known to be an automorphism on the Zemanian space $H_{\mu}\left(\mathbb{R}_{+}\right), \mathbb{R}_{+}=(0, \infty)$, consisting of all complex-valued infinitely differentiable functions $\phi$ on $\mathbb{R}_{+}$which satisfy

$$
\gamma_{m, k}^{\mu}(\phi)=\sup _{x \in \mathbb{R}_{+}}\left|x^{m}\left(x^{-1} D\right)^{k} x^{-\mu-1 / 2} \phi(x)\right|<\infty, \quad \forall m, k \in \mathbb{N}_{0}
$$

From (7), we have

$$
\begin{aligned}
& \left(B_{\theta} g\right)(b, a) \\
& =a^{2 \mu+1} b^{-\mu-1 / 2} \int_{0}^{\infty} x^{-\mu-1 / 2}(b x / a)^{1 / 2} J_{\mu}(b x / a) h_{\mu}\left(t^{\mu+1 / 2} g(t)\right)(x / a) h_{\mu}\left(z^{\mu+1 / 2} \overline{\theta(z)}\right)(x) d x \\
& =a^{\mu+3 / 2} b^{-\mu-1 / 2} \int_{0}^{\infty}(u)^{-\mu-1 / 2}(b u)^{1 / 2} J_{\mu}(b u) h_{\mu}\left(t^{\mu+1 / 2} g(t)\right)(u) h_{\mu}\left(z^{\mu+1 / 2} \overline{\theta(z)}\right)(a u) d u .
\end{aligned}
$$

Now, let us set

$$
\begin{aligned}
& u^{-\mu-1 / 2} h_{\mu}\left(t^{\mu+1 / 2} g(t)\right)=\left(h_{\mu} f\right)(u), \\
& z^{\mu+1 / 2} \overline{\theta(z)}=\overline{\psi(z)}
\end{aligned}
$$

and

$$
\left(B_{\psi} f\right)(b, a)=b^{\mu+1 / 2} a^{-\mu-3 / 2}\left(B_{\theta} g\right)(b, a)
$$

to get the following convenient form of the Bessel wavelet transform:

$$
\left(B_{\psi} f\right)(b, a):=\int_{0}^{\infty}(b u)^{1 / 2} J_{\mu}(b u) \tilde{f}(u) \overline{\tilde{\psi}(a u)} d u:=h_{\mu}(\tilde{f}(u) \overline{\tilde{\psi}(a u)})(b)
$$

where $\tilde{f}(u)=\left(h_{\mu} f\right)(u)$. 
From [6, p.134], we shall use the following Leibnitz-type formula:

$$
\left(x^{-1} D\right)^{k}\left(x^{-\mu-1 / 2} \psi \phi\right)=\sum_{s=0}^{k}\left(\begin{array}{l}
k \\
s
\end{array}\right)\left(x^{-1} D\right)^{s}\left(x^{-\mu-1 / 2} \phi\right)\left(x^{-1} D\right)^{k-s} \psi \text {. }
$$

\section{The space $H_{\mu}^{\omega}$}

Let $\omega$ be a continuous real-valued function defined on $\mathbb{R}_{+}=(0, \infty)$ such that $\omega(0)=0$ and
(a) $\quad 0 \leq \omega(u+v) \leq \omega(u)+\omega(v), \quad \forall u, v \in \mathbb{R}_{+}$,
(b) $\int_{0}^{\infty} \frac{\omega(u) d u}{1+u^{2}}<\infty$,
(c) $\quad \omega(u) \geq l+d \log (1+u)$

for some real $l$ and $d>0$.

The class of all such $\omega$ functions is denoted by $M$.

Now, assume that $\omega$ is a function in $M$. A function $f$ is said to be in the space $H_{\mu}^{\omega}$, where $f$ and $h_{\mu} f$ are smooth functions, and for every $\mu \in \mathbb{R}_{+}, n \in \mathbb{N}_{0}$ and $\mathrm{m}$ is a positive real number,

$$
\alpha_{m, n}^{\mu}(f)=\sup _{u \in \mathbb{R}_{+}} e^{m \omega(u)}\left|\left(u^{-1} D\right)^{n} u^{-\mu-1 / 2} f(u)\right|<\infty
$$

On $H_{\mu}^{\omega}$ we consider the topology generated by the family $\left\{\alpha_{m, n}^{\mu}\right\}_{m \in \mathbb{R}_{+}, n \in \mathbb{N}_{0}}$ of seminorms. From $[1,2]$, we have

$$
\beta_{m, n}^{\mu}(f)=\sup _{u \in \mathbb{R}_{+}} e^{m \omega(u)}\left|\left(u^{-1} D\right)^{n} u^{-\mu-1 / 2}\left(h_{\mu} f\right)(u)\right|<\infty, \quad \forall m \in \mathbb{R}_{+}, n \in \mathbb{N}_{0} .
$$

In what follows, we study the Bessel wavelet transform $B_{\psi}$ of infinite order on $H_{\mu}^{\omega}$. For this purpose, we define the symbol class $S^{\rho, \omega}$.

Definition 2.1 The function $\tilde{\psi}(x): C^{\infty}\left(\mathbb{R}_{+}\right) \rightarrow \mathbb{C}$ belongs to class $S^{\rho, \omega}$ if and only if $\forall m \in \mathbb{R}_{+}$

$$
e^{m \omega(x)}\left|\left(x^{-1} \partial / \partial x\right)^{p} \overline{\tilde{\psi}(x)}\right| \leq c^{p, \rho}\left(1+x^{2}\right)^{\rho-p}, \quad \forall p \in \mathbb{N}_{0}, \rho \in \mathbb{R},
$$

where $c$ is a constant and $\tilde{\psi}$ denotes the Hankel transform of the basic wavelet $\psi$.

In this section, certain spaces of functions of Beurling-type are introduced on which Bessel wavelet transforms can be defined. First, we recall the definition of the Zemanian space $H_{\mu}\left(\mathbb{R}_{+}\right)$.

Definition 2.2 The set of all infinitely differentiable functions $\left(B_{\psi} \phi\right)(b, a)$ on $\mathbb{R}_{+}^{2}$ satisfying the condition

$$
\gamma_{n, l}^{\mu, m, m^{\prime}}\left(B_{\psi} \phi\right)=\sup _{a, b} e^{m \omega(b)-m^{\prime} \omega(a)}\left|\left(b^{-1} D\right)^{n}\left(a^{-1} D\right)^{l} b^{-\mu-1 / 2}\left(B_{\psi} \phi\right)(b, a)\right|<\infty,
$$

$\forall n, l \in \mathbb{N}_{0}$, is denoted by $H_{\mu}^{\omega}\left(\mathbb{R}_{+}^{2}\right)$, where $\mu, m, m^{\prime} \in \mathbb{R}$. 
Theorem 2.1 The Bessel wavelet transform $B_{\psi} \phi$ is a continuous linear map of $H_{\mu}^{\omega}\left(\mathbb{R}_{+}\right)$ into $H_{\mu}^{\omega}\left(\mathbb{R}_{+}^{2}\right)$ for $\mu \geq-1 / 2$.

Proof To complete the proof of the theorem, we need to show that $\left(B_{\psi} \phi\right)(b, a)$ satisfies (16). From the property (b) of the function $\omega(u)$, it follows that to every $\epsilon>0$, there exists a constant $c(\epsilon)$ such that

$$
\omega(u) \leq \epsilon u+c(\epsilon)
$$

so that

$$
e^{m \omega(u)} \leq e^{m c(\epsilon)} \sum_{\nu=0}^{\infty} \frac{(m \epsilon)^{\nu}}{\nu !} u^{\nu} .
$$

Using equation (10) and the technique of Zemanian [6, p.141], we can write

$$
\begin{aligned}
& e^{m \omega(b)}\left|\left(b^{-1} D\right)^{n} b^{-\mu-1 / 2}\left(B_{\psi} \phi\right)(b, a)\right| \\
& \leq e^{m c(\epsilon)} \sum_{\nu=0}^{\infty} \frac{(m \epsilon)^{\nu}}{\nu !} b^{\nu}\left|\left(b^{-1} D\right)^{n} b^{-\mu-1 / 2}\left(B_{\psi} \phi\right)(b, a)\right| \\
& =e^{m c(\epsilon)} \sum_{\nu=0}^{\infty} \frac{(m \epsilon)^{\nu}}{\nu !} b^{\nu}\left|\left(b^{-1} D\right)^{n} b^{-\mu-1 / 2} h_{\mu}(\tilde{\phi}(u) \overline{\tilde{\psi}(a u)})\right| \\
& =e^{m c(\epsilon)} \sum_{\nu=0}^{\infty} \frac{(m \epsilon)^{\nu}}{\nu !} \\
& \times\left|\int_{0}^{\infty} u^{2 \mu+2 n+v+1}\left\{\left(u^{-1} D\right)^{v}\left(u^{-\mu-1 / 2} \overline{\tilde{\psi}(a u)} \tilde{\phi}(u)\right)\right\}(b u)^{-\mu-n} J_{\mu+v+n}(b u) d u\right| .
\end{aligned}
$$

Since for $\mu \geq-1 / 2,\left|(b u)^{-\mu-n} J_{\mu+v+n}(b u)\right|$ is bounded on $0<b, u<\infty$ by $Q_{\mu}$, the right-hand side of equation (21) can be estimated by

$$
\begin{aligned}
& \leq e^{m c(\epsilon)} Q_{\mu} \sum_{\nu=0}^{\infty} \frac{(m \epsilon)^{v}}{v !} \int_{0}^{\infty} u^{2 \mu+2 n+v+1}\left|\left(u^{-1} D\right)^{v}\left(u^{-\mu-1 / 2} \overline{\tilde{\psi}(a u)} \tilde{\phi}(u)\right)\right| d u \\
& =e^{m c(\epsilon)} Q_{\mu} \sum_{\nu=0}^{\infty} \frac{(m \epsilon)^{v}}{v !} \int_{0}^{\infty} u^{2 \mu+2 n+v+1} \\
& \quad \times\left|\sum_{r=0}^{v}\left(\begin{array}{l}
v \\
r
\end{array}\right)\left(u^{-1} D\right)^{r}\left(u^{-\mu-1 / 2} \tilde{\phi}(u)\right)\left(u^{-1} D\right)^{v-r} \overline{\tilde{\psi}(a u)}\right| d u .
\end{aligned}
$$

Therefore,

$$
\begin{aligned}
& e^{m \omega(b)}\left|\left(b^{-1} D\right)^{n}\left(a^{-1} D\right)^{l} b^{-\mu-1 / 2}\left(B_{\psi} \phi\right)(b, a)\right| \\
& \leq e^{m c(\epsilon)} Q_{\mu} \sum_{\nu=0}^{\infty} \frac{(m \epsilon)^{v}}{\nu !} \int_{0}^{\infty} u^{2 \mu+2 n+v+1} \sum_{r=0}^{\nu}\left(\begin{array}{l}
\nu \\
r
\end{array}\right) \\
& \quad \times\left|\left(u^{-1} D\right)^{\nu-r}\left(u^{-\mu-1 / 2} \tilde{\phi}(u)\right)\right|\left|\left(u^{-1} D\right)^{r}\left(a^{-1} D\right)^{l} \tilde{\tilde{\psi}(a u)}\right| d u .
\end{aligned}
$$


In view of estimate (17), we have for $t=a u$

$$
\begin{aligned}
& \left|\left(u^{-1} D\right)^{r}\left(a^{-1} D\right)^{l} \overline{\tilde{\psi}(a u)}\right| \\
& \quad=\left|a^{2 r} u^{2 l}\left(t^{-1} d / d t\right)^{r+l} \overline{\tilde{\psi}(t)}\right| \\
& \quad \leq c^{r+l, \rho}\left(1+t^{2}\right)^{\rho-r-l} a^{2 r} u^{2 l} \\
& \quad=c^{r+l, \rho}\left(1+a^{2} u^{2}\right)^{\rho-r-l} a^{2 r} u^{2 l} .
\end{aligned}
$$

Therefore, (22) becomes

$$
\begin{aligned}
& e^{m \omega(b)}\left|\left(b^{-1} D\right)^{n}\left(a^{-1} D\right)^{l} b^{-\mu-1 / 2}\left(B_{\psi} \phi\right)(b, a)\right| \\
& \leq e^{m c(\epsilon)} Q_{\mu} \sum_{\nu=0}^{\infty} \frac{(m \epsilon)^{\nu}}{\nu !} \int_{0}^{\infty} u^{2 \mu+2 n+v+1} \\
& \quad \times \sum_{r=0}^{v}\left(\begin{array}{l}
v \\
r
\end{array}\right)\left|\left(u^{-1} D\right)^{\nu-r}\left(u^{-\mu-1 / 2} \tilde{\phi}(u)\right)\right| c^{r+l, \rho}\left(1+a^{2} u^{2}\right)^{\rho-r-l} a^{2 r} u^{2 l} d u \\
& \leq e^{m c(\epsilon)} Q_{\mu} \sum_{\nu=0}^{\infty} \frac{(m \epsilon)^{\nu}}{\nu !} \sum_{r=0}^{v}\left(\begin{array}{l}
v \\
r
\end{array}\right) c^{r+l, \rho}\left(1+a^{2}\right)^{\rho-r-l}\left(1+a^{2}\right)^{r} \\
& \quad \times \int_{0}^{\infty} u^{2 \mu+2 n+v+1+2 l}\left(1+u^{2}\right)^{\rho-r-l}\left|\left(u^{-1} D\right)^{\nu-r}\left(u^{-\mu-1 / 2} \tilde{\phi}(u)\right)\right| d u .
\end{aligned}
$$

Suppose $P$ is an integer not less than $2 \mu+2 n+2 l+1$, then

$$
u^{2 \mu+2 n+v+1+2 l} \leq(1+u)^{P+v} .
$$

Using (25), the right-hand side of equation (24) can be bounded by

$$
\begin{aligned}
e^{m c(\epsilon)} & Q_{\mu} \sum_{\nu=0}^{\infty} \sum_{r=0}^{v}\left(\begin{array}{l}
\nu \\
r
\end{array}\right) c^{r+l, \rho} \frac{(m \epsilon)^{\nu}}{\nu !}(1+a)^{2(\rho-l)} \\
& \times \int_{0}^{\infty}(1+u)^{P+\nu}(1+u)^{2(\rho-r-l)}\left|\left(u^{-1} D\right)^{\nu-r}\left(u^{-\mu-1 / 2} \tilde{\phi}(u)\right)\right| d u \\
= & e^{m c(\epsilon)} Q_{\mu}(1+a)^{2(\rho-l)} \sum_{\nu=0}^{\infty} \sum_{r=0}^{v}\left(\begin{array}{l}
v \\
r
\end{array}\right) c^{r+l, \rho} \frac{(m \epsilon)^{\nu}}{\nu !} \\
& \times \int_{0}^{\infty}(1+u)^{P+\nu+2 \rho}(1+u)^{-2 l}\left|\left(u^{-1} D\right)^{\nu-r}\left(u^{-\mu-1 / 2} \tilde{\phi}(u)\right)\right| d u .
\end{aligned}
$$

Using inequality (14), the above expression is bounded by

$$
\begin{aligned}
& e^{m c(\epsilon)} Q_{\mu, l, \rho}^{\prime} e^{-2(\rho-l) l / d} e^{2(\rho-l) \omega(a) / d} \sum_{\nu=0}^{\infty} \sum_{r=0}^{v}\left(\begin{array}{l}
v \\
r
\end{array}\right) c^{r} \frac{(m \epsilon)^{\nu}}{\nu !} e^{-(P+2 \rho+v) l / d} \\
& \times \sup _{u \in \mathbb{R}_{+}} e^{((P+2 \rho+v) / d) \omega(u)}\left|\left(u^{-1} D\right)^{\nu-r}\left(u^{-\mu-1 / 2} \tilde{\phi}(u)\right)\right| \int_{0}^{\infty} \frac{d u}{(1+u)^{2 l}} .
\end{aligned}
$$


Using property (16), we can estimate the right-hand side of (26) by

$$
\begin{aligned}
& e^{m c(\epsilon)} Q_{\mu, l, \rho}^{\prime} e^{-(P+4 \rho-2 l) l l d} e^{2(\rho-l) \omega(a) / d} \sum_{\nu=0}^{\infty} \sum_{r=0}^{\nu}\left(\begin{array}{c}
\nu \\
r
\end{array}\right) c^{r} \frac{(m \epsilon)^{\nu}}{\nu !} e^{-(\nu l) / d} \beta_{(P+2 \rho+\nu) / d,(\nu-r)}^{\mu}(\phi) \\
&= Q_{\mu, l, \rho}^{\prime} e^{2(\rho-l) \omega(a) / d} \exp (m c(\epsilon)-(P+4 \rho-2 l) l / d) \sum_{\nu=0}^{\infty} \frac{\left((m \epsilon) e^{-l / d}\right)^{\nu}}{\nu !}(1+c)^{\nu} \\
& \times \max _{0 \leq r \leq \nu}\left[\left\{\beta_{(P+2 \rho+\nu) / d,(\nu-r)}^{\mu}(\phi)\right\}^{1 / \nu}\right]^{\nu} \\
&= Q_{\mu, l, \rho}^{\prime} e^{2(\rho-l) \omega(a) / d} \exp (m c(\epsilon)-(P+4 \rho-2 l) l / d) \sum_{\nu=0}^{\infty} \frac{\left((m \epsilon) e^{-l / d}(1+c)\right)^{\nu}}{\nu !} \\
& \quad \times \max _{0 \leq r \leq \nu}\left[\left\{\beta_{(P+2 \rho+\nu) / d,(\nu-r)}^{\mu}(\phi)\right\}^{1 / \nu}\right]^{\nu} .
\end{aligned}
$$

Therefore,

$$
\begin{aligned}
& e^{m \omega(b)-m^{\prime} \omega(a)}\left|\left(b^{-1} D\right)^{n}\left(a^{-1} D\right)^{l} b^{-\mu-1 / 2}\left(B_{\psi} \phi\right)(b, a)\right| \\
& \leq Q_{\mu, l, \rho}^{\prime} \exp (m c(\epsilon)-(P+4 \rho-2 l) l / d) \\
& \quad \times \sum_{\nu=0}^{\infty} \frac{\left((m \epsilon) e^{-l / d}(1+c)\right)^{\nu}}{\nu !}\left[\max _{0 \leq r \leq \nu}\left\{\beta_{(P+2 \rho+v) / d,(\nu-r)}^{\mu}(\phi)\right\}^{1 / \nu}\right]^{\nu},
\end{aligned}
$$

where $m^{\prime}=2(\rho-l) / d$. Now, choosing

$$
\epsilon<\left\{\max _{0 \leq r \leq v} \beta_{(P+2 \rho+v) / d,(v-r)}^{\mu}(\phi)\right\}^{-1 / v}\left(\nu ! m e^{-l / d}(1+c)\right)^{-1},
$$

we find that the last series is convergent. Therefore,

$$
e^{m \omega(b)-m^{\prime} \omega(a)}\left|\left(b^{-1} D\right)^{n}\left(a^{-1} D\right)^{l} b^{-\mu-1 / 2}\left(B_{\psi} \phi\right)(b, a)\right|<\infty .
$$

Hence, $\left(B_{\psi} \phi\right) \in H_{\mu}^{\omega}\left(\mathbb{R}_{+}^{2}\right)$.

\section{The space $G_{\mu}^{\omega}$}

The Bessel-differential operator $S_{\mu}$ is defined by

$$
S_{\mu, x}=\frac{d^{2}}{d x^{2}}+\frac{\left(1-4 \mu^{2}\right)}{4 x^{2}} .
$$

From [6, p.139] we know that for any $\phi \in H_{\mu}\left(\mathbb{R}_{+}\right)$,

$$
h_{\mu}\left(S_{\mu} \phi\right)=-y^{2} h_{\mu} \phi
$$

and

$$
S_{\mu, x}^{r} \phi(x)=\sum_{j=0}^{r} c_{j} x^{2 j+\mu+1 / 2}\left(x^{-1} D\right)^{r+j}\left(x^{-\mu-1 / 2} \phi(x)\right),
$$

where $c_{j}$ are constants depending only on $\mu$. 
Now, assume that $\omega$ is a function in $M$. $A$ function $\phi \in C^{\infty}\left(\mathbb{R}_{+}\right)$is said to be in the space $G_{\mu}^{\omega}$ if for every $\mu \in \mathbb{R}, n \in \mathbb{N}_{0}$, and $m$ is a positive real number,

$$
A_{m, n}^{\mu}(\phi)=\sup _{x \in\left(\mathbb{R}_{+}\right)} e^{m \omega(x)}\left|S_{\mu}^{n} \phi(x)\right|<\infty .
$$

The family $\left\{A_{m, n}^{\mu}\right\}_{m \in \mathbb{R}, n \in \mathbb{N}_{0}}$ of seminorms generates the topology of $G_{\mu}^{\omega}$.

Definition 3.1 The set of all infinitely differentiable functions $\left(B_{\psi} \phi\right)(b, a)$ on $\mathbb{R}_{+}^{2}$ satisfying the condition

$$
\delta_{n}^{\mu, m, m^{\prime}}\left(B_{\psi} \phi\right)=\sup _{a, b} e^{m \omega(b)-m^{\prime} \omega(a)}\left|S_{\mu, b}^{n}\left(B_{\psi} \phi\right)(b, a)\right|<\infty, \quad \forall n \in \mathbb{N}_{0},
$$

is denoted by $G_{\mu}^{\omega}\left(\mathbb{R}_{+}^{2}\right)$, where $\mu, m, m^{\prime} \in \mathbb{R}$.

Theorem 3.1 The Bessel wavelet transform $B_{\psi}$ is a continuous linear map of $G_{\mu}^{\omega}\left(\mathbb{R}_{+}\right)$into $G_{\mu}^{\omega}\left(\mathbb{R}_{+}^{2}\right)$ for $\mu \geq-1 / 2$.

Proof As in the proof of Theorem 2.1, using inequality (21), we have

$$
\begin{aligned}
& e^{m \omega(b)}\left|S_{\mu, b}^{n}\left(B_{\psi} \phi\right)(b, a)\right| \\
& \quad \leq e^{m c(\epsilon)} \sum_{\nu=0}^{\infty} \frac{(m \epsilon)^{\nu}}{\nu !} b^{\nu} \sum_{j=0}^{n}\left|c_{j}\right| b^{2 j+\mu+1 / 2}\left|\left(b^{-1} D\right)^{n+j} b^{-\mu-1 / 2}\left(B_{\psi} \phi\right)(b, a)\right| \\
& \quad=e^{m c(\epsilon)} \sum_{\nu=0}^{\infty} \sum_{j=0}^{n}\left|c_{j}\right| \frac{(m \epsilon)^{\nu}}{\nu !} b^{2 j+\nu+\mu+1 / 2}\left|\left(b^{-1} D\right)^{n+j} b^{-\mu-1 / 2}\left(B_{\psi} \phi\right)(b, a)\right| .
\end{aligned}
$$

Assume that $0 \leq \mu+1 / 2<p$, where $p$ is a positive integer, then $b^{\mu+1 / 2} \leq(1+b)^{\mu+1 / 2} \leq$ $(1+b)^{p}$, and the right-hand side of equation (31) is bounded by

$$
\begin{aligned}
e^{m c(\epsilon)} & \sum_{\nu=0}^{\infty} \sum_{j=0}^{n}\left|c_{j}\right| \frac{(m \epsilon)^{\nu}}{\nu !}(1+b)^{2 j+v+p}\left|\left(b^{-1} D\right)^{n+j} b^{-\mu-1 / 2}\left(B_{\psi} \phi\right)(b, a)\right| \\
= & e^{m c(\epsilon)} \sum_{\nu=0}^{\infty} \sum_{j=0}^{n}\left|c_{j}\right| \frac{(m \epsilon)^{\nu}}{\nu !} \sum_{k=0}^{2 j+v+p}\left(\begin{array}{c}
2 j+\nu+p \\
k
\end{array}\right) b^{k} \\
& \times\left|\left(b^{-1} D\right)^{n+j} b^{-\mu-1 / 2} h_{\mu}(\overline{\tilde{\psi}(a u)} \tilde{\phi}(u))(b)\right| .
\end{aligned}
$$

Using Zemanian's technique, equation (10) and the Leibnitz-type formula (11), the last expression can be estimated by

$$
\begin{aligned}
e^{m c(\epsilon)} & \sum_{v=0}^{\infty} \sum_{j=0}^{n}\left|c_{j}\right| \frac{(m \epsilon)^{\nu}}{\nu !} \sum_{k=0}^{2 j+v+p}\left(\begin{array}{c}
2 j+v+p \\
k
\end{array}\right) \\
\quad \times & \mid \int_{0}^{\infty} u^{2 \mu+2(n+j)+k+1}\left(u^{-1} D\right)^{k}\left(u^{-\mu-1 / 2} \overline{\tilde{\psi}(a u)} \tilde{\phi}(u)\right) \\
& \times(b u)^{-(\mu+n+j)} J_{\mu+k+(n+j)}(b u) d u \mid
\end{aligned}
$$




$$
\begin{aligned}
\leq & e^{m c(\epsilon)} Q_{\mu} \sum_{\nu=0}^{\infty} \sum_{j=0}^{n} \sum_{k=0}^{2 j+v+p}\left|c_{j}\right| \frac{(m \epsilon)^{\nu}}{\nu !}\left(\begin{array}{c}
2 j+v+p \\
k
\end{array}\right) \\
& \times \int_{0}^{\infty} u^{2 \mu+2(n+j)+k+1} \sum_{s=0}^{k}\left(\begin{array}{l}
k \\
s
\end{array}\right)\left|\left(u^{-1} D\right)^{k-s}\left(u^{-\mu-1 / 2} \tilde{\phi}(u)\right)\right|\left|\left(u^{-1} D\right)^{s} \bar{\psi} \tilde{\psi}(a u)\right| d u .
\end{aligned}
$$

In view of estimate (17), the above expression can be bounded by

$$
\begin{aligned}
& e^{m c(\epsilon)} Q_{\mu} \sum_{v=0}^{\infty} \sum_{j=0}^{n} \sum_{k=0}^{2 j+v+p}\left|c_{j}\right| \frac{(m \epsilon)^{\nu}}{v !}\left(\begin{array}{c}
2 j+v+p \\
k
\end{array}\right) \int_{0}^{\infty} u^{2 \mu+2(n+j)+k+1} \\
& \times \sum_{s=0}^{k}\left(\begin{array}{l}
k \\
s
\end{array}\right)\left|\left(u^{-1} D\right)^{k-s}\left(u^{-\mu-1 / 2} \tilde{\phi}(u)\right)\right| a^{2 s} c^{s, \rho}\left(1+a^{2} u^{2}\right)^{\rho-s} d u \\
& \leq e^{m c(\epsilon)} Q_{\mu} \sum_{v=0}^{\infty} \sum_{j=0}^{n} \sum_{k=0}^{2 j+v+p}\left|c_{j}\right| \frac{(m \epsilon)^{v}}{v !}\left(\begin{array}{c}
2 j+v+p \\
k
\end{array}\right) \int_{0}^{\infty} u^{2 \mu+2(n+j)+k+1} \\
& \quad \times \sum_{s=0}^{k}\left(\begin{array}{l}
k \\
s
\end{array}\right)\left|\left(u^{-1} D\right)^{k-s}\left(u^{-\mu-1 / 2} \tilde{\phi}(u)\right)\right|\left(1+a^{2}\right)^{s} c^{s, \rho}\left(1+a^{2}\right)^{\rho-s}\left(1+u^{2}\right)^{\rho-s} d u \\
& \leq e^{m c(\epsilon)} Q_{\mu} \sum_{v=0}^{\infty} \sum_{j=0}^{n} \sum_{k=0}^{2 j+v+p} \sum_{s=0}^{k}\left|c_{j}\right| \frac{(m \epsilon)^{\nu}}{v !}\left(\begin{array}{c}
2 j+v+p \\
k
\end{array}\right)\left(\begin{array}{l}
k \\
s
\end{array}\right)\left(1+a^{2}\right)^{2 \rho} c^{s, \rho} \\
& \quad \times \int_{0}^{\infty} u^{2 \mu+2(n+j)+k+1}(1+u)^{2(\rho-s)}\left|\left(u^{-1} D\right)^{k-s}\left(u^{-\mu-1 / 2} \tilde{\phi}(u)\right)\right| d u .
\end{aligned}
$$

Suppose that $N$ is a positive integer not less than $2 \mu+6 n+p+1$, then the above expression can be estimated by

$$
\begin{gathered}
e^{m c(\epsilon)} Q_{\mu} \sum_{v=0}^{\infty} \sum_{j=0}^{n} \sum_{k=0}^{2 j+v+p} \sum_{s=0}^{k}\left|c_{j}\right| \frac{(m \epsilon)^{\nu}}{\nu !}\left(\begin{array}{c}
2 j+v+p \\
k
\end{array}\right)\left(\begin{array}{l}
k \\
s
\end{array}\right) c^{s, \rho}(1+a)^{2 \rho} \\
\times \int_{0}^{\infty}(1+u)^{N+v}(1+u)^{2(\rho-s)}\left|\left(u^{-1} D\right)^{k-s}\left(u^{-\mu-1 / 2} \tilde{\phi}(u)\right)\right| d u .
\end{gathered}
$$

Using the inequality $(1+u) \leq e^{-l / d} e^{\omega(u) / d}$ from (14), the right-hand side of the above expression can be bounded by

$$
\begin{aligned}
& e^{m c(\epsilon)} Q_{\mu, \rho}^{\prime} \sum_{\nu=0}^{\infty} \sum_{j=0}^{n} \sum_{k=0}^{2 j+v+p} \sum_{s=0}^{k}\left|c_{j}\right| \frac{(m \epsilon)^{v}}{\nu !}\left(\begin{array}{c}
2 j+v+p \\
k
\end{array}\right)\left(\begin{array}{l}
k \\
s
\end{array}\right) c^{s} e^{-(2 \rho l) / d} e^{(2 \rho \omega(a)) / d} e^{-(N+v+2 \rho) l / d} \\
& \quad \times \sup _{u \in \mathbb{R}_{+}} e^{(N+v+2 \rho) \omega(u) / d}\left|\left(u^{-1} D\right)^{k-s}\left(u^{-\mu-1 / 2} \tilde{\phi}(u)\right)\right| \int_{0}^{\infty} \frac{d u}{(1+u)^{2 s}}
\end{aligned}
$$

Using property (16), we can estimate the right-hand side of (32) by

$$
\begin{aligned}
e^{m c(\epsilon)} & Q_{\mu, \rho}^{\prime} e^{-(N+4 \rho) l / d} e^{(2 \rho \omega(a)) / d} \sum_{\nu=0}^{\infty} \sum_{k=0}^{2 j+v+p} \sum_{j=0}^{n}\left(\begin{array}{c}
2 j+v+p \\
k
\end{array}\right)\left|c_{j}\right| \frac{(m \epsilon)^{\nu}}{\nu !} e^{-l v / d}(1+c)^{k} \\
& \times \max _{0 \leq s \leq k} \beta_{(N+v+2 \rho) / d,(k-s)}^{\mu}(\phi)
\end{aligned}
$$




$$
\begin{aligned}
= & e^{(2 \rho \omega(a)) / d} Q_{\mu, \rho}^{\prime} \exp (m c(\epsilon)-(N+4 \rho) l / d) \sum_{\nu=0}^{\infty} \sum_{k=0}^{2 j+v+p} \sum_{j=0}^{n}\left(\begin{array}{c}
2 j+v+p \\
k
\end{array}\right)\left|c_{j}\right| \frac{\left\{(m \epsilon) e^{-l / d}\right\}^{v}}{\nu !} \\
& \times\left[\left\{(1+c)^{k} \max _{0 \leq s \leq k} \beta_{(N+v+2 \rho) / d,(k-s)}^{\mu}(\phi)\right\}^{1 / \nu}\right]^{v} \cdot
\end{aligned}
$$

Therefore,

$$
\begin{aligned}
& e^{m \omega(b)-m^{\prime} \omega(a)}\left|S_{\mu, b}^{n}\left(B_{\psi} \phi\right)(b, a)\right| \\
& \leq Q_{\mu, \rho}^{\prime} \exp (m c(\epsilon)-(N+4 \rho) l / d) \sum_{\nu=0}^{\infty} \sum_{k=0}^{2 j+v+p} \sum_{j=0}^{n}\left(\begin{array}{c}
2 j+v+p \\
k
\end{array}\right)\left|c_{j}\right| \\
& \quad \times \frac{\left\{(m \epsilon) e^{-l / d}\right\}^{\nu}}{\nu !}\left[\left\{(1+c)^{k} \max _{0 \leq s \leq k} \beta_{(N+v+2 \rho) / d,(k-s)}^{\mu}(\phi)\right\}^{1 / \nu}\right]^{\nu}<\infty
\end{aligned}
$$

as the infinite series can be made convergent by choosing

$$
\epsilon<\left\{(1+c)^{k} \max _{0 \leq s \leq k} \beta_{(N+v+2 \rho) / d,(k-s)}^{\mu}(\phi)\right\}^{-1 / v}\left(\nu ! m e^{-l / d}\right)^{-1},
$$

where $m^{\prime}=2 \rho / d$.

This completes the proof.

\section{Competing interests}

The authors declare that they have no competing interests.

\section{Authors' contributions}

AP has identified the problems and suggested the solution, AM participated in the proof of the Theorems and MMD participated in the solution to find the Bessel wavelet transform. All authors read and approved the final manuscript.

\section{Author details}

'Department of Applied Mathematics, Indian School of Mines, Dhanbad, 826004, India. ${ }^{2}$ Department of Mathematics, NERIST, Nirjuli, India.

\section{Acknowledgements}

This work is supported by Indian School of Mines, Dhanbad, under grant No. 613002 /ISM JRF/Acad/2009.

Received: 17 November 2012 Accepted: 8 January 2013 Published: 22 January 2013

\section{References}

1. Belhadj, M, Betancor, Jj: Hankel transformation and Hankel convolution of tempered distributions. Rocky Mt. J. Math. 3(4), 1171-1203 (2001)

2. Pathak, RS, Shrestha, KK: The Hankel transform of Gevrey ultradistributions. Integral Transforms Spec. Funct. 11(1), 61-72 (2001)

3. Pathak, RS, Prasad, A: The pseudo-differential operator $h_{\mu, a}$ on some Gevrey spaces. Indian J. Pure Appl. Math. 34(8), 1225-1236 (2003)

4. Haimo, DT: Integral equations associated with Hankel convolutions. Trans. Am. Math. Soc. 116, 330-375 (1965)

5. Pathak, RS, Dixit, MM: Bessel wavelet transform on certain function and distribution spaces. J. Anal. Appl. 1,65-83 (2003)

6. Zemanian, AH: Generalized Integral Transformations. Interscience, New York (1968)

doi:10.1186/1029-242X-2013-29

Cite this article as: Prasad et al.: Continuity of the Bessel wavelet transform on certain Beurling-type function spaces. Journal of Inequalities and Applications 2013 2013:29. 